\title{
Moving ethical regulation forward
}

\author{
Disciplinary divides and diverse views on the role of ethical review in the social and behavioural sciences \\ shape interpretations of the recent Common Rule changes. Challenges lay ahead in creating a shared \\ standard for all those engaged in research that involves human subjects.
}

Regulations, like laws created by statute, are subject to interpretation. The Common Rule, the policy regulating federally funded research involving human subjects in the United States, is no exception. Recent, long-awaited revisions to the Common Rule are currently being lauded for both their deregulatory spirit and simultaneously criticized for their incoherence. Ultimately, the impact of the revisions will be determined by how institutions conducting research with human subjects interpret and implement the new rules.

First published in 1991, the Common Rule provides for institutional review boards (IRBs) to review research involving human participants, establishes requirements for consent, and sets forth policies for assuring compliance. The regulation does not cover non-federally funded research, but through Federalwide Assurance (FWA), institutions are invited to voluntarily extend the Common Rule regulations to non-federally funded human-subjects research. FWAs are held by domestic and foreign institutions alike, and historically the Common Rule has influenced ethical regulation worldwide.

On 19 January 2017, the Office for Human Research Protections (OHRP) within the Department of Health and Human Services (DHHS) issued final revisions to the Common Rule. The revisions are the first major changes to this regulatory framework in nearly 25 years. The new regulation was long awaited after five and a half years of development and more than 3,300 public comments. It has now entered into an implementation preparation period and relevant parties have until January 2018 to come into compliance.

When the DHHS began its regulatory review effort in 2011, it noted a need to address the effectiveness and efficiency of the existing regulation in light of changing research practices and technologies.

Included among developments in the research environment was an increase in social science research. The final revision continues to assert the OHRP's power to regulate the humanities and social sciences, and the changes in the final revision largely reflect these broader goals. Exactly how far the changes go to reconcile regulatory practice with contemporary research practice is debatable.

Three articles in this issue of Nature Human Behaviour shed light on what was and what was not accomplished with revisions to the Common Rule as it pertains to the regulation of social and behavioural science research. Susan Fiske and Jeanne Rivard (article number 0091) laud the revisions as a new era in humansubjects research regulation. Under the revised Common Rule, most ordinary social and behavioural science research is exempt from IRB review - including "benign behavioral interventions" that describe most ordinary psychology experiments - exemption determinations need not be approved by IRBs, consent is now required to be communicated clearly, and vulnerable populations have been redefined to include only subjects with impaired decision-making capabilities.

\section{The impact of the revisions will be determined by how institutions conducting research with human subjects interpret and implement the new rules.}

The revisions are not without detractors, however. Robert Dingwall (article number 0083) argues that the final revisions maintain decades of misguided practice and overreach by IRBs. While some scholarly activities, including journalism, history and legal research, may now escape IRB review provisioned by the Common Rule, the work of anthropologists and sociologists conducting participant observation or ethnographic studies gets no such reprieve. Rejecting the proposed category of 'excluded', failing to establish a clear distinction between registration for purposes of exemption determination and exemption from review, and creating a new process of "limited IRB review" does little to actually improve the efficiency and effectiveness of ethical governance. Dingwall argues that the revisions are incoherent and the Common Rule remains a regulatory framework intended for the biomedical sciences and superimposed upon a set of disciplines with a markedly different set of concerns.

Notably, one rationale for modernizing the regulations was the development of technologies that have changed the way data is collected, analysed and shared. For disciplines where physical harm may no longer top the list, breaches of privacy and confidentiality have emerged as a key concern in the era of big data. The final revision of the Common Rule, however, mostly fails to address this issue. The language of clinical trials and biospecimen safeguards is inadequate for dealing with the vast amounts of digital data collected from mobile devices, social media and commercial transactions that are not easily deidentified, says Julia Lane (article number 0075). Rather than wait for the Common Rule regulations to catch up to research practice, Lane calls the community to action - develop a standard set of privacy practices and usage protections that are relevant to contemporary social and behavioural science.

The revisions to the Common Rule do move ethical regulation of the social and behavioural sciences forward, even if much more modestly than hoped for. Clearly, they do not satisfy all researchers or address all of the concerns of a diverse community of scholars. The differing interpretations of the revisions in this issue highlight the difficulties in creating a uniform standard for all those engaged in human-subjects research. Finding the right regulatory approach that both protects human subjects and enables efforts to fully understand the human condition will remain a challenge as the research environment continues to change at a pace far faster than the regulatory apparatus. 